\title{
Altitudinal visual field asymmetry is coupled with altered retinal circulation in patients with normal pressure glaucoma
}

\author{
Oliver Arend, Andreas Remky, Louis B Cantor, Alon Harris
}

\begin{abstract}
Aim-To compare the effect of altitudinal asymmetric glaucomatous damage on retinal microcirculation in patients with normal pressure glaucoma (NPG).

Methods-In a prospective cross sectional study patients with NPG (washed out for antiglaucomatous therapy) and altitudinal asymmetric perimetric findings between the superior and inferior hemisphere (Humphrey 24-2) $(n=18)$ were included and compared with 20 NPG patients with symmetrical field defects and 18 healthy subjects. Fluorescein angiograms were performed using a scanning laser ophthalmoscope. Using digital image analysis, arteriovenous passage time (AVP) and vessel diameters were assessed for comparison of corresponding affected and less affected temporal arcades.
\end{abstract}

Results-Both affected and less affected hemispheres showed significantly prolonged AVP times $(p<0.001)$ when compared with healthy subject data. In hemispheres with more severe glaucomatous field loss the AVP times were significantly $(p=0.04)$ prolonged compared with the less affected hemisphere (AVP affected 3.1 (SD 7) seconds $v$ AVP less affected 2.61 (1.4) seconds). There was no asymmetry effect on arterial and venous diameter measurements.

Conclusion-Altitudinal visual field defects are linked together with circulatory deficits of the retinal tissue. The attenuated circulation seems to be a considerable factor in the natural course of glaucomatous optic neuropathy.

(Br f Ophthalmol 2000;84:1008-1012)

In the pathogenesis of glaucomatous neuropathy, particularly in normal pressure glaucoma (NPG), disturbances of ocular circulation seem important in the natural course of the disease. Different techniques ${ }^{1-7}$ are employed to assess vascular dysfunction. Fluorescein angiography has been shown to detect ischaemic lesions of the optic disc. ${ }^{89}$ Circulatory fluorescein angiographic studies showed abnormalities of retinal and choroidal circulation in patients with chronic open angle or normal tension glaucoma. ${ }^{31011}$ This may be attributed to the effect of decreased perfusion pressure due to elevated intraocular pressure, also a considerable factor since arteriovenous passage (AVP) was prolonged despite antiglaucomatous treatment. ${ }^{3}$ However, the correlation of the AVP to the degree of glaucomatous field damage has not yet been examined.

Early glaucomatous visual field defects are often asymmetric showing either superior or inferior nerve fibre bundle defects. ${ }^{12}$ Furthermore, particularly in NPG, eccentric cupping corresponds well with typical arcuate defects. ${ }^{12}$ Fluorescein angiography allows measurements of retinal circulation corresponding to visual hemifield defects. Past studies correlating visual function and microcirculation provided important comparative asymmetric data among both eyes on macular ${ }^{1}$ or retrobulbar circulation. ${ }^{13} 14$ In this study, we examined retinal microcirculation in patients with NPG and the correlation with altitudinal asymmetric visual field loss. This approach was chosen because a direct comparison of glaucomatous visual field defect and corresponding retinal microcirculation is possible. From existing data both a nonassociation or association of altered retinal circulation seems possible and the results could underline the significance of such measurement.

\section{Materials and methods}

Scanning laser video fluorescein angiograms (SLO; Rodenstock Institute, Ottobrunn, Germany) combined with an image analysis system (Matrox Inspector, Matrox Graphics, Quebec, Canada) allow the evaluation of AVP times and corresponding arterial and venous diameters. The method has been presented in detail elsewhere. ${ }^{15}$ In short, fluorescein angiograms at 40 degree field mode $\left(29^{\circ} \times 23^{\circ}\right)$ were performed. Dynamic sequences $(25 \mathrm{~Hz})$ were analysed using an image processing unit in order to measure the first appearance of the dye in a retinal artery and the first influx in the corresponding vein by dye dilution technique. Measurements were performed in the temporal superior and temporal inferior vessel formation. The AVP time characterises the shortest passage time through the vascular segment from the artery, via the capillary bed, to the corresponding veins. ${ }^{15}$ Past studies have shown that measurements of AVP times are reproducible and of clinical significance. ${ }^{3} 1617$

At the location of the selected points for AVP measurements the arterial and venous diameters were quantified from densitometric curves by using a "half height of maximum height" procedure. ${ }^{18} 19$ The measurement location of the AVP and diameter measurement was situated half to one disc diameter away from the disc margin on the major temporal arteries and veins. Diameter measurements were performed in mid-transit fluorescein pic- 
A
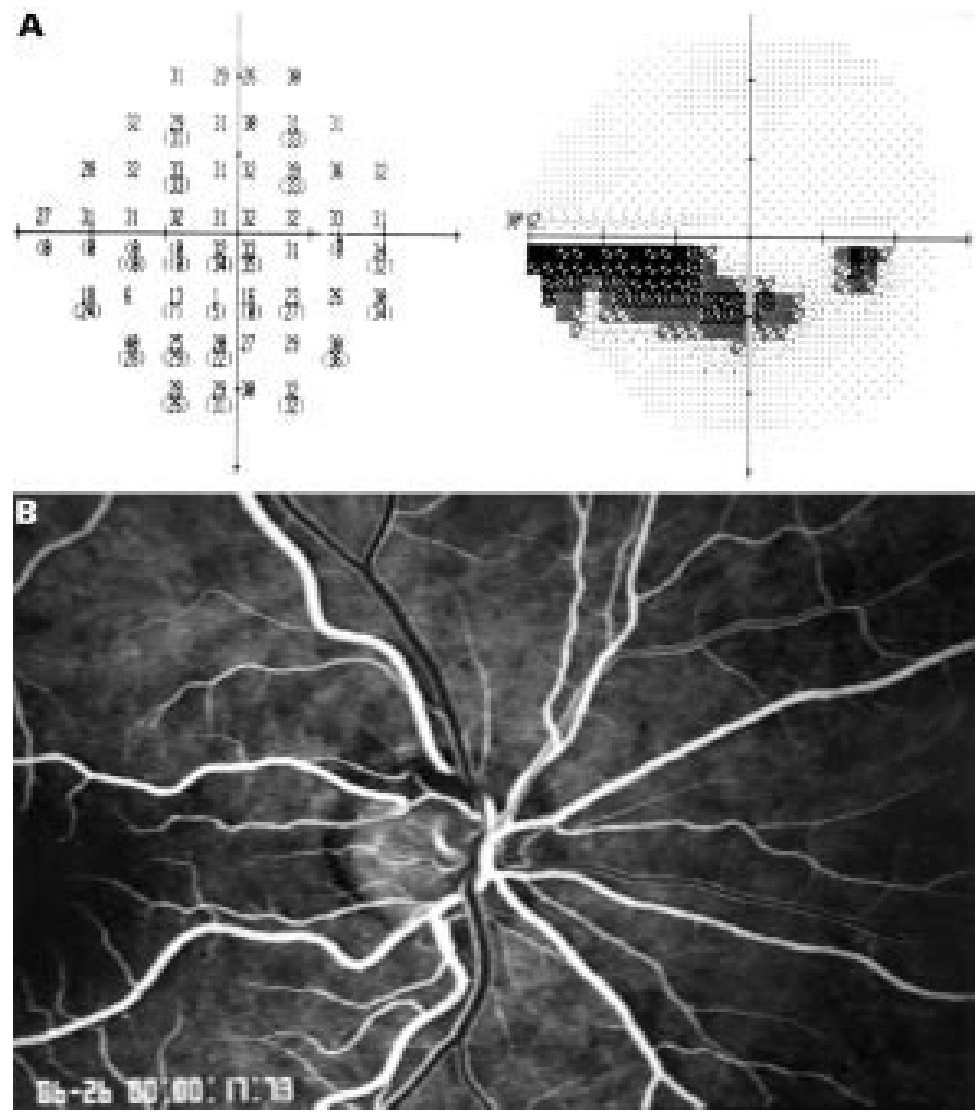

Figure 1 (A) Asymmetric visual field loss of the inferior visual field (Humphrey 24-2) with an arcuate scotoma in the inferior hemifield. (B) Corresponding fluorescein angiogram with focal ischaemia of the optic disc temporally superior (AVP time of the affected temporal superior vessel: 2.17 seconds ; the less affected temporal inferior vessel formation: 1.67 seconds).

tures $(768 \times 512$ pixels $)$ of the vessel in the $20^{\circ}$ observation mode $\left(15^{\circ} \times 14^{\circ}\right)$. The width of the measuring profile was 5 pixels and to generate subpixel accuracy the average of five repeated measurements is given.

The scanning laser ophthalmoscope allows the choice of automatic video gain control, which is confounding for dye dilution curve analysis. Therefore, the video gain which was calibrated linearly was kept at a constant level. For all the angiograms the argon laser illumination was kept at a constant level (level 6). All data were corrected for individual magnification error by using axial length, determined by A-scan ultrasonography for computation of the improved Littmann formula. ${ }^{20}{ }^{21}$ Dynamic and morphological analyses were performed in a masked fashion without prior knowledge of visual field or optic nerve head asymmetry.

Visual fields were performed with a Humphrey field analyser (program 24-2) and interpreted on the basis of two comparable fields. Significant asymmetric damage was considered if the superior or inferior hemifield differed in stage of glaucomatous damage (modified from Aulhorn and Karnemeyer ${ }^{22}$ - stage $0=$ no relative scotomata; stage $1=$ relative scotomata; stage $2=$ dense accurate scotomata without connection to the optic disc; stage $3=$ dense accurate scotomata with connection to the optic disc; stage $4=$ complete loss of a hemifield; stage $5=$ loss of the central visual field-only small temporal eccentric field remaining). Altitudinal visual field asymmetry was present in patients who showed different stages of the visual field score (Aulhorn criteria) when comparing the lower and upper hemispheres. Visual fields were classified without knowledge of the patient's name and circulatory results. For statistical comparison of affected versus less affected visual hemifields all given mean defects calculated by the STATPAC single point analysis were divided with the number of measured points for each hemifield. The clinical examinations included intraocular pressure (IOP) measurements by Goldmann tonometry. The given IOP data represent measurements without antiglaucomatous therapy. Blood pressure and heart rate were measured with an automatic device (Dinap Criticare Vital Daten Monitor, Criticare, Tampa, FL, USA).

\section{PATIENTS}

Prospectively, in this study 38 normal pressure glaucoma (NPG) patients (intraocular pressure $\leqslant 21 \mathrm{~mm} \mathrm{Hg}$ ) were recruited and 18 patients showed asymmetric visual field loss qualifying for further analysis. The topical antiglaucomatous medications of all patients were washed out for 3 weeks before examination. Figures 1 and 2 show asymmetric visual field loss and the corresponding fluorescein angiogram. The diagnosis was based on repeated IOP measurements (profile including midnight measurements), glaucomatous optic nerve head cupping (stereoscopic examination and argon laser imaging (SLO)), and visual field defects (Humphrey Instruments) as well as open angles (gonioscopy). ${ }^{23}$ Exclusion criteria included history of allergy to fluorescein and a refractive error $\geqslant-8$ dioptres The lens density was judged from standardised retroilluminated charts (LOCS III $^{24}$ ) and only subjects with mild nuclear colour changes (NC 1-2) were included.

Informed consent was obtained from each subject including detailed explanations of all procedures before participation in this study. The protocol for the study was reviewed and approved by the institutional review board of the Indiana University, Indianapolis, USA. The tenets of the Helsinki declaration were followed throughout the study.

In 18 patients (seven male, 11 female) asymmetric visual field damage was recorded. The mean visual acuity was $20 / 25$. The field was defined as being asymmetric if the hemifields had different stages according to the classification by Aulhorn and Karnemeyer. ${ }^{22}$ The number of patients assigned to stages were: stage 1 three, stage 2 eight, stage 3 one, and stage 4 six. Twelve patients $(66 \%)$ had asymmetric loss in the superior visual field and six patients $(33 \%)$ in the inferior visual field. The standard visual field indices $(\mathrm{MD}=$ mean deviation, PSD = pattern standard deviation, $\mathrm{MT}=$ mean threshold) were derived from Humphrey 24-2 full threshold fields $(\mathrm{MD}=$ $-7.3(\mathrm{SD} 4.5) \mathrm{dB} ; \mathrm{PSD}=9.1(4.8) \mathrm{dB} ; \mathrm{MT}=$ $21.8(4.2) \mathrm{dB})$.

In 20 patients with NPG (six male, 14 female) no altitudinal defects were detected 

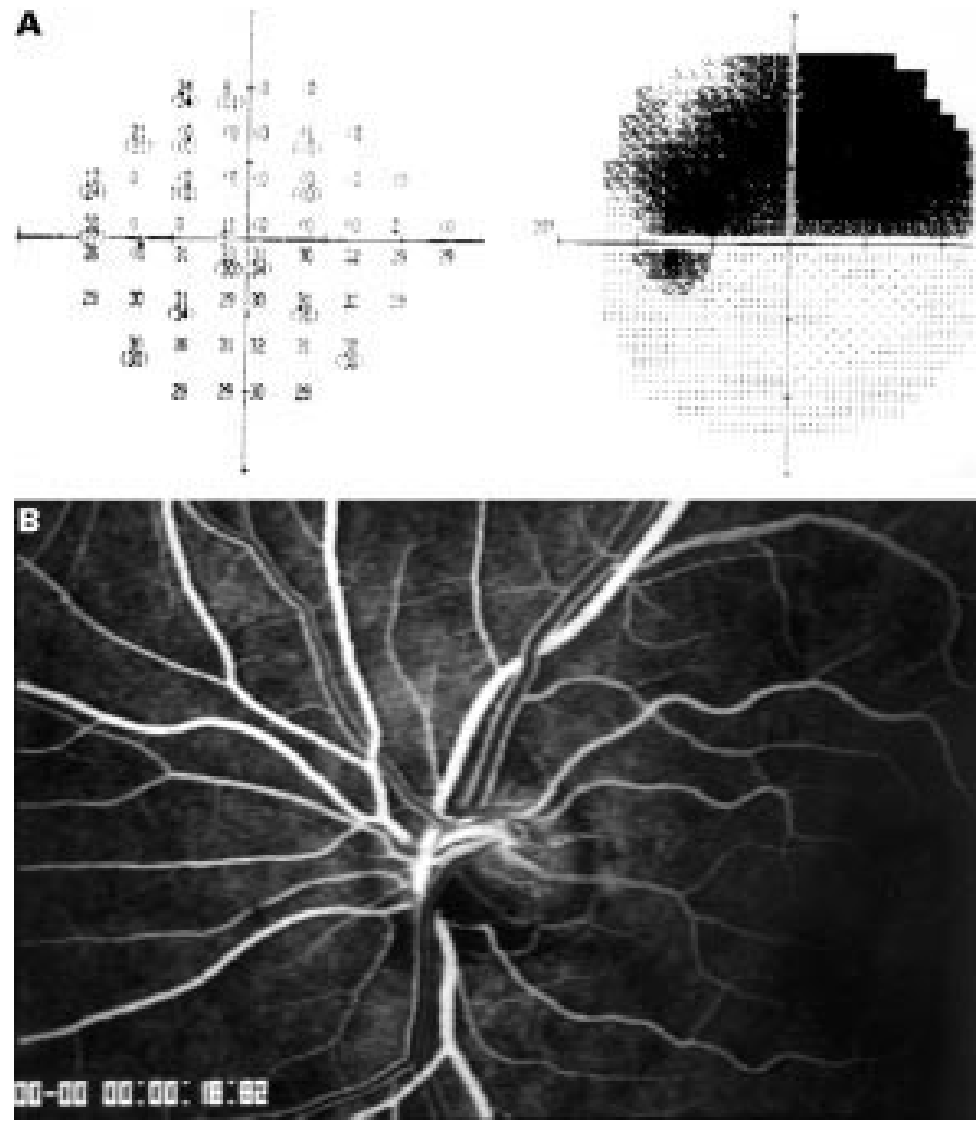

Figure 2 (A) Asymmetric hemifield visual field loss of the superior visual field (Humphrey 24-2). (B) Corresponding fuorescein angiogram with ischaemia of the inferior portion of the optic disc (AVP of affected inferior vessel formation: 3.6 seconds; AVP of less affected superior vessel formation: 2.23 seconds). Note the visible different fluorescein intensity of the laminar stream of the dye in the veins superiorly and inferiorly.

Table 1 Clinical and demographic data (mean (SD)) for the normal pressure glaucoma (NPG) patients with and without asymmetric visual field loss

\begin{tabular}{|c|c|c|c|c|}
\hline & $\begin{array}{l}\text { Healthy } \\
\text { subjects }\end{array}$ & $\begin{array}{l}\text { Asymmetric } \\
\text { NPG patients }\end{array}$ & $\begin{array}{l}\text { Symmetric } \\
\text { NPG patients }\end{array}$ & Significance ${ }^{\star}$ \\
\hline No (male/female) & $18(8 / 10)$ & $18(7 / 11)$ & $20(6 / 14)$ & $\mathrm{p}=0.65$ \\
\hline Age (years) & $54(15)$ & $60(15)$ & $60(13)$ & $\mathrm{p}=0.37$ \\
\hline Systolic blood pressure $(\mathrm{mm} \mathrm{Hg})$ & $135(21)$ & $123(17)$ & $119(12)$ & $\mathrm{p}=0.11$ \\
\hline \multicolumn{5}{|l|}{ Diastolic blood pressure } \\
\hline$(\mathrm{mm} \mathrm{Hg})$ & $75(14)$ & $74(9)$ & $73(8)$ & $\mathrm{p}=0.62$ \\
\hline Intraocular pressure $(\mathrm{mm} \mathrm{Hg})$ & $16(2)$ & $17(4)$ & $16(3)$ & $\mathrm{p}=0.97$ \\
\hline Heart rate (beats/s) & $77(13)$ & $80(12)$ & $71(12)$ & $\mathrm{p}=0.33$ \\
\hline
\end{tabular}

^Kruskal-Wallis test and $\chi^{2}$ test.

among visual fields. The standard visual field indices were as following: $\mathrm{MD}=-3.7$ (3.9) $\mathrm{dB} ; \mathrm{PSD}=5.3(3.9) \mathrm{dB} ; \mathrm{MT}=23.6(4.2) \mathrm{dB}$. The healthy control group consisted of 18 subjects (eight male/10 female). The mean visual acuity was $20 / 20$ and there was no evidence or history of any ocular or systemic disease.

STATISTICAL ANALYSIS

Mean value and standard deviation are given for all samples with normal distributions
(Kolmogorov-Smirnov test) and non-normal distributions median and percentiles $(10 \%$ and $90 \%$ ). The paired Wilcoxon signed rank test was used to assess the significance of the differences between paired groups and the MannWhitney $\mathrm{U}$ test for unpaired comparisons. For multiple paired group comparisons the Friedman test was used and for unpaired group comparisons the Kruskall-Wallis test was used. Findings smaller than 0.05 were considered to be statistically significant. Pearson correlation coefficients were calculated to evaluate the relation between the variables. $p$ Values were obtained after carrying out Fisher's $r$ to $\mathrm{z}$ transformations.

\section{Results}

The clinical and demographic data of the examined groups (NPG patients with and without altitudinal field asymmetry) did not differ significantly (Table 1 ). The average AVP times (mean of the superior and inferior vessel formation) were significantly prolonged (asymmetric NPG: $p<0.001$; symmetric NPG: $\mathrm{p}<0.001$ ) when compared with healthy subject data.

In NPG patients with altitudinal asymmetric visual fields the mean deviation of the affected hemifield differed significantly from the less affected (Table 2). The AVP time in the affected field was significantly prolonged $(12 \%)$ whereas there was no difference of arterial or venous vessel diameter (Table 3 ).

In patients with NPG and symmetric field defects the mean deviation of the superior hemifield differed not significantly from the inferior (Table 2). These patients showed no significant differences between the temporal superior and inferior vessel formation for the AVP time and the arterial and venous diameters (Table 4). Additionally, no asymmetry effect between temporal superior and inferior vessels was observed for healthy subjects for AVP times and vessel diameters (Table 5).

The average AVP time of temporal superior and inferior vessel formation in the asymmetric NPG group showed no significant correlation with intraocular pressure, diastolic, or systolic blood pressure.

\section{Discussion}

A variety of circulatory disturbances are known in normal pressure glaucoma patients. ${ }^{25}{ }^{26} \mathrm{Cur}-$ rent concepts describe an energy deficit as the possible key for the initiation of a cytotoxic cascade. ${ }^{27}$ If an ischaemic change is thought to be causative ${ }^{28} 29$ the knowledge of the microcirculation may be important. Although the laminar and prelaminar region of the optic nerve is the area of major damage ${ }^{30}$ no existing method for microcirculatory quantification allows di-

Table 2 Mean deviation of affected and less affected hemifields in patients with altitudinal asymmetric field defects are presented (median, 10, 90\% percentiles). Furthermore the mean defects of the temporal superior and inferior field of NPG with symmetric field defects are given (median, $10,90 \%$ percentiles)

\begin{tabular}{lllll}
\hline & $\begin{array}{l}\text { Mean deviation affected } \\
\text { branch }(d B)\end{array}$ & $\begin{array}{l}\text { Mean deviation less } \\
\text { affected branch }(d B)\end{array}$ & $\begin{array}{l}\text { Mean deviation } \\
\text { superior field }(d B)\end{array}$ & $\begin{array}{l}\text { Mean deviation } \\
\text { inferior field }(d B)\end{array}$ \\
\hline $\begin{array}{l}\text { NPG with altitudinal asymmetric field defects } \\
\text { NPG with symmetric field defects }\end{array}$ & $-9.1(-25.3 ;-3.2)$ & $-1.7(-6.9 ; 0.5)$ & $-4.1(-15.1 ; 0.5)$ & $-3.4(-15.4 ; 0.4)$ \\
\hline
\end{tabular}


Table 3 Arteriovenous passage times (AVP) and corresponding arterial and venous vessel diameters of the affected and less affected vessel formations (altitudinal visual field loss) (mean $(S D))$

\begin{tabular}{llll}
\hline$n=18$ & AVP (seconds) & Arterial diameter $(\mu m)$ & Venous diameter $(\mu m)$ \\
\hline Affected branch & $3.1(1.67)$ & $99(19)$ & $135(28)$ \\
Less affected branch & $2.61(1.44)$ & $101(23)$ & $140(30)$ \\
Significance $^{\star}$ & $\mathrm{p}=0.04$ & $\mathrm{p}=0.89$ & $\mathrm{p}=0.87$ \\
\hline
\end{tabular}

$\star$ Wilcoxon signed rank test.

Table 4 Arteriovenous passage times (AVP) and corresponding arterial and venous vessel diameters of the temporal superior and inferior vessel formation in NPG patients without altitudinal visual field defects (mean (SD))

\begin{tabular}{llll}
\hline$n=20$ & AVP $($ seconds $)$ & Arterial diameter $(\mu m)$ & Venous diameter $(\mu m)$ \\
\hline Temporal superior & $2.80(1.60)$ & $97(20)$ & $130(22)$ \\
Temporal inferior & $2.65(1.33)$ & $106(18)$ & $143(26)$ \\
Significance $^{\star}$ & $\mathrm{p}=0.66$ & $\mathrm{p}=0.12$ & $\mathrm{p}=0.17$ \\
\hline
\end{tabular}

ॠWilcoxon signed rank test.

Table 5 Arteriovenous passage times (AVP) and corresponding arterial and venous vessel diameters of the temporal superior and inferior vessel formation in healthy subjects (mean (SD))

\begin{tabular}{llll}
\hline$n=18$ & AVP $($ seconds) & Arterial diameter $(\mu m)$ & Venous diameter $(\mu m)$ \\
\hline Temporal superior & $1.57(0.40)$ & $95(9)$ & $132(18)$ \\
Temporal inferior & $1.62(0.37)$ & $98(9)$ & $127(21)$ \\
Significance $^{\star}$ & $\mathrm{p}=0.24$ & $\mathrm{p}=0.45$ & $\mathrm{p}=0.44$ \\
\hline
\end{tabular}

^Wilcoxon signed rank test.

rect evaluation of in vivo results. Retinal measurements appear of interest especially since nerve fibre haemorrhages are coupled with disease progression, and retinal nerve fibre loss is a characteristic feature of glaucomatous optic neuropathy particularly in patients with NPG. ${ }^{31}$

In the presented study, the retinal arteriovenous passage (AVP) time of NPG patients is almost doubled compared with healthy subject data, indicating a severe attenuation of retinal circulation. These results are in agreement with previous studies $^{317}$ in patients with chronic open angle glaucoma demonstrating prolonged AVP times and decreased mean dye velocities. The question arises, whether prolonged AVP times correspond with decreased perfusion or malnutrition of the tissue. Theoretically, a vaso-occlusive disease could result via decreased resistance and arteriovenous shunts in shortened AVP times. In patients with diabetic angiopathy AVP is prolonged with increasing severity of retinopathy. ${ }^{32}$ Furthermore, in NPG patients no retinal capillary closure has been detected. ${ }^{33}$ Thus, AVP measurements representing the passage through from the artery passing the capillary formation to the vein are an indicator of the integrity and regulation of a vascular segment. The presented results are in agreement with other investigations revealing significantly decreased retrobulbar circulation ${ }^{43}$ and pulsatile ocular blood flow ${ }^{6}$ in NPG patients when compared with healthy subjects.

Studies of visual field asymmetry ${ }^{35}$ have demonstrated considerable differences in NPG and high tension glaucoma suggesting different pathogenic mechanisms. Additionally, higher intraocular pressure is not necessarily associated with more severe visual field loss or a major cause resulting in asymmetric field damage. ${ }^{35}{ }^{36}$ The comparison of psychophysical and circulatory data might provide information about the pathophysiology - for example, the question whether or not circulatory deficits may be a factor in disease progression.

In patients with ocular hypertension and primary open angle glaucoma blue field entoptic simulation demonstrated a significantly decreased macular leucocyte velocity in the more severely affected eye when performing binocular asymmetry analysis. ${ }^{1}$ In patients with primary open angle glaucoma, laser Doppler flowmetry showed decreased perfusion of the optic nerve head and juxtapapillary retina in patients with no or only borderline visual field defects. ${ }^{5}$ NPG patients with asymmetric visual field damage have decreased retrobulbar velocities in the more affected eye $^{13}$ and the decrease in retrobulbar velocities was correlated with progressive visual field loss. ${ }^{14}$ Pulsatile ocular blood flow is reduced in NPG patients, regardless of the visual field loss when compared with healthy subjects, and significantly lower in the eye with more severe field damage. ${ }^{6}$ These circulatory studies did not account for regional differences of the superior and inferior portion of the optic nerve head or the corresponding retinal vasculature.

This study comparing superior and inferior altitudinal damage in one eye allows direct quantification of the affected circulatory bed versus the less affected one. In patients with NPG and altitudinal visual field loss the retinal AVP time as measured in the corresponding temporal superior or inferior vessel formation was significantly prolonged. In contrast, NPG patients with symmetric field defects, or healthy subjects, showed no circulatory asymmetry among superior and inferior vessel formations.

The effect observed in this study might represent a secondary effect due to the loss of nerve fibres and the downregulation due to reduced metabolic needs. ${ }^{37}$ However, the retinal segment of the less affected visual field also showed significantly prolonged AVP times compared with healthy subjects. Thus, a circulatory deficit might represent an early insult in glaucomatous disease. ${ }^{6}$ However, the primary cause remains unclear when visual field integrity does not necessarily reflect nerve fibre health. ${ }^{38}$

Descending neuropathies (for example, retinitis pigmentosa) are typically associated with reduction of vessel diameters attributed to a metabolic downregulation. The arterial and venous diameter data in this study did not provide any significant association with altitudinal field loss. Past studies ${ }^{39}$ in patients with chronic open angle glaucoma showed that vessel diameters were correlated with visual field indices. The differences might be explained by the range of visual field data of the included patients; in other words, by including patients with severe field loss one would expect more pronounced narrowing of the vessel diameters. Those patients might be more affected by a downregulation as mentioned above. Thus, in this study with only moderate visual field loss, no significant alterations of vessel diameters were detected compared with the less affected hemifield. 
In conclusion, circulatory deficits of the retinal tissue are linked together with visual field loss. Both the affected and less affected branches show prolonged AVP times when compared with healthy subjects. Circulatory changes should be considered in the treatment regimen when the cascade of events leading to loss of visual function is most amenable to being interrupted.

Proprietary interest: none.

Presented in part at the annual meeting of the American Academy 1998 (New Orleans, USA).

This investigation was supported by Start 4/96-4 (OA, AR), and in part by NIH Grant EY 10801 and Research to Prevent Blindness (IU) (AH).

1 Sponsel WE, DePaul KL, Kaufman PL. Correlation of visual function and retinal leukocyte velocity in glaucoma. Am f Ophthalmol 1990;

2 Grunwald JE, Riva CE, Stone RA, et al. Retinal autoregulation in open-angle glaucoma. Ophthalmology 1984;91: 1690-4.

3 Wolf S, Arend O, Sponsel WE, et al. Retinal hemodynamics using scanning laser ophthalmoscopy and hemorheology in chronic open-angle glaucoma. Ophthalmology 1993;100: 1561-6.

4 Rojanapongpun P, Drance SM, Morrison BJ. Ophthalmic artery flow velocity in glaucomatc

5 Michelson G, Langhans MJ, Harazny J, et al. Visual field in the neuroretinal rim area in primary open angle glaucoma. Graefes Arch Clin Exp Ophthalmol 1998;236:80-5.

6 Fontana L, Poinoosawmy D, Bunce CV, et al. Pulsatile ocular blood flow investigation in asymmetric normal tension glaucoma and normal subjects. Br f Ophthalmol 1998;82: glauco

7 Rankin SJA, Walman BE, Buckley AR, et al. Color Doppler maging and spectral analysis of the optic nerve vasculature in glaucoma. Am f Ophthalmol 1995;119:685-93.

8 Spaeth GL. Fluorescein angiography: its contributions towards understanding the mechanisms of visual loss in glaucoma. Trans Am Ophthalmol Soc 1975;73:491-553.

9 Schwartz B. Circulatory defects of the optic disk and retina in ocular hypertension and high pressure open-angle glaucoma. Surv Ophthalmol 1994;38(Suppl):23-34.

10 Suzuki R, Sugihara I, Kurimoto S. Retinal circulation in primary open angle glaucoma tested by videodensitometric primary open angle glaucoma tested by videodensi

11 Richard G, Hackelbusch R, Schmidt KU, et al. Untersuchung zur Haemodynamik des Auges bei Glaucoma chronicum sinplex und low-tension Glaucom-eine videoangiographische Studie. Fortschr Ophthalmol 1988;85:369-72.
Weber J. Topographie der funktionellen Schädigung beim Weber J. Topographie der funktionellen Schädigung bein

3 Nicolela M, Drance SM, Rankin SA, et al. Color Doppler Nicolela M, Drance SM, Rankin SA, et al. Color Doppler
imaging in patients with asymmetric glaucoma and unilatimaging in patients with asymmetric glaucoma and unilat-

14 Yamazaki Y, Drance SM. The relationship between progression of visual field defects and retrobulbar circulation in patients with glaucoma. Am f Ophthalmol 1997;124:28795.

15 Arend O, Harris A, Martin BJ, et al. Scanning laser ophthalmoscopy based evaluation of epipapillary velocities:method and physiological variability. Surv Ophthalmol 1999;

44(Suppl 1):3-9.
16 Wolf S, Arend O, Reim M. Measurement of retinal hemodynamics with scanning laser ophthalmoscopy: reference values and variation. Surv Ophthalmol (Suppl) 1994;38:95100 .
17 Wolf S, Arend O, Haase A, et al. Retinal hemodynamics in patients with chronic open angle glaucoma. Germ $f$ Ophthalmol 1995;4:279-82.

18 Delori FC, Fitch KA, Feke GT, et al. Evaluation of micrometric and microdensitometric methods for measuring the width of retinal vessel images on fundus photographs. Graefes Arch Clin Exp Ophthalmol 1988;226: 393-9.

19 Remky A, Arend O, Beausencourt E, et al. Retinale Gefässe vor und nach Photokoagulation bei diabetischer Retinopathie: Durchmesserbestimmungen mittels digitalisierter Farbdiapositive. Klin Monatsbl Augenheilkd 1996; 207:79-83.

20 Littmann H. Zur Bestimmung der wahren Größe eines Objektes auf dem Hintergrund eines lebenden Auges. Klin Monatsbl Augenheilkd 1988;192:66-7.

21 Bennett AG, Rudnicka AR, Edgar DF. Improvements on Littmann's method of determining the size of retinal features by fundus photography. Graefes Arch Clin Exp Ophthalmol 1994;232:361-7.

22 Aulhorn E, Karnemeyer H. Frequency distribution in early glaucomatous visual field defects. Doc Ophthalmol Proc Ser 1977;14:75-83.

23 Werner EB. Normal-tension glaucoma. In: Ritch R, Shields $\mathrm{MB}$, Krupin T, eds. The glaucomas. 2nd ed. St Louis: Mosby, 1996:769-97.

24 Chylack CTJ, Wolfe JK, Singer DM, et al. The lens opacities classification system III. The longitudinal sudy of the cataract study group. Arch Ophthalmol 1993;111:831-6.

25 Phelps CD, Corbett JJ. Migraine and low tension glaucoma. Invest Ophthalmol Vis Sci 1985;26:1105-8.

26 Drance SM, Douglas GR, Wijsman K, et al. Response of blood flow to warm and cold in normal and low-tension glaucoma patients. Am f Ophthalmol 1988;105:35-9.

27 Caprioli J. Neuroprotection of the optic nerve in glaucoma. Acta Ophthalmol Scand 1997;75:364-7.

28 Tomita H, Ishiguro S, Tamai M. Apoptosis in the ischemic injury and the change of low affinity NGF receptor expression in the rat retinas after ischemia and reperfusion. Invest Ophthalmol Vis Sci 1996;37:144 (abstract).

29 Kobayashi N, Nishokawa S, Ishiguro S, et al. Immunoreactivities of glutamate and GABA in the rat retinas after ischemia. Invest Ophthalmol Vis Sci 1996;37:145 (abstract).

30 Quigley HA, Addicks EM. Regional differences in the structure of the lamina cribrosa and their relation to glaucomatous optic nerve damage. Arch Ophthalmol 1981;99:13743.

31 Siegner S, Netland P. Optic disc hemorrhages and progression of glaucoma. Ophthalmology 1996;103:1014-24.

32 Bertram B, Wolf S, Fiehöfer S, et al. etinal circulation times in diabetes mellitus type I. Br F Ophthalmol 1991;75:462-5.

33 Arend O, Remky A, Harris A. Prolonged retinal circulation is coupled with unaffected macular capillary density in patients with normal tension glaucoma. Invest Ophthalmol Vis Sci 1999;40:508.

34 Vecsei PV, Hommer A, Reitner A, et al. Farbduplex der retrobulbären Arterien bei Normaldruck- und Offenwinkelglaukom. Klin Monatsbl Augenheilkd 1998;212:444-8.

35 Poinoosawmy D, Fontana L, Wu JX, et al. Frequency of asymmetric visual field defects in normal tension and hightension glaucoma. Ophthalmology 1998;105:988-91.

36 Jonas J, Gründler AE, Gonzales-Cortes J. Pressuredependent neuroretinal rim loss in normal tension glaucoma. Am f Ophthalmol 1998;125:137-44.

37 Anderson DR. Glaucoma, capillaries and pericystes. 1. Blood flow regulation. Ophthalmologica 1996;210:257-62. 38 Frisen FM. A simple relationship between the probability distribution of visual acuity and the density of retinal output channels. Acta Ophthalmol 1976;54:437-44.

39 Jonas J, Nguyen XN, Naumann GOH. Parapapillary retinal vessel diameter in normal and glaucoma eyes. Invest Ophthalmol Vis Sci 1989;30:1599-603. 